\title{
EJEMPLO DE INTEGRACIÓN DE ALEXA CON UN ROBOT UR
}

\author{
J.M. Vicente-Samper, I. Campos, B. Sanz, A. Rodriguez, J. M. Oñate, J. M. Sabater-Navarro \\ Grupo de Neuroingeniería Biomédica, Universidad Miguel Hernández de Elche
}

\begin{abstract}
Resumen
Este trabajo muestra la solución adoptada por un grupo de estudiantes de robótica para permitir una interacción natural con un robot haciendo uso de un altavoz inteligente y de los servicios interactivos en nube. Esta solución permite integrar dentro de un robot industrial opciones avanzadas de interacción con voz o gestión de BBDD con visualización remota de variables del robot. Se muestra el esquema general y detalles de la implementación software.
\end{abstract}

Palabras clave: Alexa, interacción con voz, interfaz inteligente.

\section{INTRODUCCION}

En la actualidad, una de las opciones para simplificar las complejas estructuras de navegación en las interfaces hombre-máquina pasa por utilizar asistentes virtuales con inteligencia artificial, capaces de interpretar los comandos de una forma natural, [1]. Estos asistentes virtuales son capaces no solo de recibir y enviar información en forma de voz, sino que también pueden trabajar con bases de datos en tiempo real, lo que permite que sea adaptable a cualquier entorno de trabajo o aplicación.

La automatización industrial desarrolla a finales del siglo XX la línea de investigación denominada Control Inteligente. Esta línea trata de sumar a los procesos de control existentes la inteligencia artificial (IA) permitiendo una interacción de alto nivel con el proceso industrial a controlar [2]

El crecimiento global de la demanda de servicios en la nube es cada vez mayor. Son muchas las empresas que migran su trabajo de centros de datos locales a computación en la nube. Según la consultora Gartner, en el año 2025 , el $80 \%$ de las organizaciones del mundo lo habrán hecho [3]. Este modelo de prestación de servicios en la nube permite operar de una forma más rápida y eficiente, escalar recursos fácilmente ante el crecimiento de la demanda o responder ante ésta de forma flexible y adaptativa. Las principales compañías del mercado de los servicios de computación en la nube actualmente son Microsoft [4], AWS [5], Dell EMC [6] e IBM [7].
Todas ellas ofrecen variedad de servicios (Internet de las cosas - IoT, bases de datos, máquinas virtuales, soportes de ejecución, hosting, etc) que se adaptan al cliente, permitiéndoles ligar todo el desarrollo de los proyectos en una única plataforma.

Este trabajo presenta una solución basada en un asistente virtual basado en computación en la nube para el control de un robot UR3e. Para ello se muestra las soluciones adoptadas por un grupo de estudiantes dentro del concurso hackathon 2019 organizado por la empresa Universal Robot. Se muestra la implementación con Alexa de Amazon de una interfaz de reconocimiento de voz que hace uso de la base de datos DynamoDB para comunicarse con el robot UR3e. Finalmente se muestra un ejemplo de interacción con el robot.

\section{MATERIALES Y MÉTODOS}

\subsection{EL ROBOT UR3}

Atendiendo a la definición comercial dada por la empresa fabricante del robot UR3, un cobot es un robot colaborativo capaz de tener una interacción física y directa con una persona. Gracias a la detección segura de colisión $\mathrm{y}$ fuerza, $\mathrm{y}$ otras medidas activas y pasivas de reducción de riesgo, se evita la obligación de instalar jaulas de protección y así la facilitación del uso compartido en el espacio entre robot-humano.

El robot de menor tamaño y carga útil de la gama de Universal Robots, el UR3e, con un rango de $500 \mathrm{~mm}$ y una carga útil de $3 \mathrm{~kg}$, es el elegido para este ejemplo de integración. Entre sus características físicas cabe destacar sus 6 grados de libertad y su muñeca no esférica. En comparación con su pariente anterior, el UR3-CB, la frecuencia de intercambio de datos varía de una forma significativa de $125 \mathrm{~Hz}$ a $500 \mathrm{~Hz}$.

\subsubsection{Comunicaciones}

Las opciones de programación del robot UR3e son:

- Interfaz gráfica de usuario/máquina por medio de Polyscope implementada a una pantalla táctil de 12 pulgadas. 
- URCap, un archivo portable construido principalmente en Java, Java Swing/HTML, y URScript, lenguaje de programación específico de Universal Robots.

En este trabajo se escoge un URCap debido a la ampliación de posibilidades que ofrece la programación en base software, dado que el propósito es la creación de una simbiosis entre un asistente personal $y$ un robot colaborativo aprovechando sus sinergias, y no una mera unión eléctrica entre inputs y outputs.

En cuanto a la comunicación entre Alexa (la nube) y el UR3e se puede realizar a través de:

- Sockets: concepto con el que dos programas, usualmente en dos dispositivos distintos definidos como cliente/servidor por dos direcciones IP, local y remota, intercambian paquetes de datos a una velocidad constante definida por la frecuencia.

- RPC: siglas en inglés para llamada a un procedimiento remoto. Es una técnica para la comunicación cliente/servidor, que consiste en invocar un programa alojado en otra computadora distinta a la local.

Esta última es la elegida para el estudio de este ejemplo dado su adaptabilidad a los lenguajes de alto nivel, integrado en el código del URCap. Y es el responsable de convocar el archivo Python en la que está alojada la llamada a la nube mediante DynamoDB.

\subsubsection{Material adicional}

El efector final del UR3e es una pinza de la marca Gimatic ${ }^{\circledR}$, modelo KIT-UR-G.

En el reto 3, explicado posteriormente en el punto 3.3, se hace uso de una cámara logitech c270 implantada entre la última articulación del cobot y el efector final, mediante un adaptador diseñado en Autodesk Inventor y prototipada por impresión 3D.

\subsection{HERRAMIENTAS DE AMAZON}

En este apartado se presentan los servicios en la nube de la plataforma de Amazon Web Services que han sido utilizados para el desarrollo de la aplicación.

\subsubsection{Alexa}

Alexa es el servicio de voz ubicado en la nube de Amazon Web Service (AWS), disponible en los dispositivos de Amazon y dispositivos de terceros con Alexa integrada [8]. Es posible desarrollar funcionalidades para Alexa, conocidas como Skill, con la herramienta Alexa Skills Kit (ASK) [9]. Se trata de un conjunto de herramientas, documentación y API en self-service que facilita el desarrollo de nuevas Skills de manera sencilla. Es posible crear una cuenta de desarrollador de manera gratuita para tener acceso a las plataformas de desarrollo.

Por otro lado, el módulo de control del servicio de voz, Alexa Voice Service (AVS) [10], encargado de ejecutar y gestionar las conversaciones entre el usuario y Alexa, se encuentra alojado en los servicios de computación en la nube de AWS, y es posible integrarlo en dispositivos comerciales de terceros como puede ser desde una Raspberry Pi hasta un vehículo.

\subsubsection{DynamoDB}

Amazon DynamoDB es un servicio de base de datos NoSQL que pertenece a AWS [11]. Admite modelos de clave-valor y de documentos, disponiendo de un esquema flexible que permite a cada fila tener cualquier número de columnas, permitiendo adaptar las tablas en un determinado momento a nuevos requisitos sin necesidad de redefinir el esquema al completo como así ocurre en bases de datos relacionales. Cada tabla posee una clave principal que facilita la indexación de la tabla. Esta puede estar formada por una clave de partición, en cuyo caso no podrá haber dos elementos que tengan el mismo valor de clave de partición o, por una clave de partición y una clave de ordenación (clave principal compuesta), donde si podrán existir diferentes elementos con una mima clave de partición, pero sus claves de ordenación deberán ser distintas. Para utilizar los servicios de AWS es necesario crear una cuenta. Además, para tener acceso de manera externa a los servicios, desde consola, scripts de ejecución, etc, será necesario instalar credenciales de la cuenta de AWS en el dispositivo desde el que se vaya a acceder a los servicios.

\subsubsection{Lambda}

AWS Lambda es un servicio de informática sin servidor que ejecuta código en respuesta a eventos y administra automáticamente los recursos informáticos, incluido el mantenimiento del servidor y del sistema operativo, aprovisionamiento de capacidad y escalado automático [12]. Ejecuta el código, denominado función de Lambda, en una infraestructura informática de alta disponibilidad. Una vez se crea la función, esta siempre está lista para ejecutarse. Cada función incluye el código y unos parámetros de configuración asociada, como la política de uso de la función. Para ello es necesario definir unos roles de ejecución y acceso a diferentes servicios, como pueden ser permisos de lectura o escritura en tablas de DynamoDB. Las funciones Lambda se utilizan como endpoint en las Skills de 
Alexa y serán las encargadas de realizar las tareas asociadas a la conversación.

\section{$2.3 \quad$ METODOS}

En este apartado se presenta el esquema de trabajo que se ha empleado como solución en la aplicación del presente artículo.

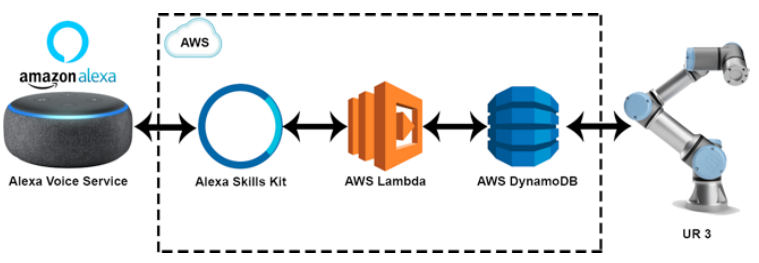

Figura 1: Esquema de trabajo

En primer lugar, el altavoz inteligente es controlado por voz. Será el encargado directo de mantener las conversaciones de voz con el usuario. Este dispositivo podrá ser cualquier sistema que posea AVS integrado, no necesariamente los dispositivos comerciales de Amazon.

El altavoz inteligente está conectado al módulo ASK de la cuenta del usuario, donde se encuentran activadas las Skills desarrolladas para la ejecución de las tareas deseadas. Dentro del módulo ASK se encuentra definido el front-end de la Skill, que mediante el uso de Intents (métodos que definen las acciones que se permiten al usuario), define el camino que debe seguir la conversación con el usuario.

Cada una de las Skills posee un endpoint o cadena de conexión a una función Lambda que hace las veces de back-end. Ésta será la encargada de realizar las tareas asociadas a la Skill de forma encubierta al usuario. Las funciones Lambda permiten realizar cambios en otros servicios de computación en la nube de AWS o invocar código utilizando llamadas a APIs externas por medio de los SDK de AWS.

La solución que se plantea en este esquema de trabajo es modificar el valor de distintas variables de una o más tablas en una base de datos de DynamoDB a través de la función Lambda. La variable a modificar y el nuevo valor vendrán determinados por el flujo en la conversación del usuario con el dispositivo Alexa, es decir, dependiendo del Intent al que acceda la Skill a través del resultado de la conversación.

Por último, un dispositivo externo, como pueda ser la controladora de un robot, se encuentra realizando un sondeo o polling constante en las variables definidas en la basde de DynamoDB. Cuando alguna de esas variables modifique su valor a través de la interacción por voz, el dispositivo externo interpretará de una manera predefinida el cambio y realizará la acción previamente programada, por ejemplo, el movimiento de un brazo robótico o la configuración de éste. Para tener acceso a la base de datos, el dispositivo externo deberá poseer las credenciales de la cuenta de AWS donde se vaya a realizar la lectura. Se ha optado por esta morfología de trabajo para aislar la ejecución del dispositivo externo de la ejecución de la función Lambda, lo que permite substituir de manera sencilla el dispositivo externo sin necesidad de variar el esquema de la Skill y de la función Lambda. Además, evita la incorporación de APIs externas en la función Lambda, lo que haría un código más complejo y pesado.

\section{EJEMPLO DE APLICACIÓN}

Estudiantes de robótica de la Universidad Miguel Hernández participaron en el Hackathon organizado por Universal Robot. En este evento se tenían que llevar a cabo 3 retos utilizando un UR3e y proponiendo soluciones para reconocimiento de voz, datamining y realidad aumentada.

\section{$3.1 \quad$ RETO 1}

El primero de ellos consistía en el manejo del robot a través de reconocimiento por voz. Aquí es donde se implementaron todas las herramientas que ofrece Alexa. A través de la plataforma AWS es posible crear y modificar una tabla de datos de dynamoDB ubicada en los servidores de Amazon, la cual está en comunicación constante con el brazo robótico. Dependiendo de los valores de la tabla el robot realizará unos movimientos u otros. Esta forma de comunicación también se aplicará en los demás retos.

La figura 2 muestra un ejemplo de interacción con la skill programada en Alexa.

\section{$3.2 \quad$ RETO 2}

El segundo reto se basa en Datamining. La aplicación principal es la visualización de datos a tiempo real. Se creó una interfaz en HTML con CSS y JavaScrip para la comunicación del robot con la tabla no MSQL. Una de las ventajas de poder usar las herramientas de Amazon es que dispones de la plataforma DynamoDB que ofrece la posibilidad de crear tablas de datos no relacionales lo que posibilita la introducción de gran cantidad de datos de cualquier tipo. 


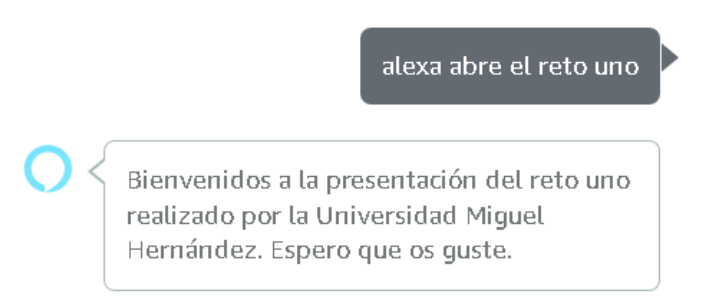

pero saluda a todos

Hola a todos. Me llamo Alexa y soy la encargada de permitir el control por voz del robot Univérsal Róbots para facilitaros su uso. Encantada de conoceros. Espero que estéis pasando una buena mañana y que estéis disfrutando de la exposición. A través de mí podréis controlar a vuestro robot desde un sofá. ¡Venga!, que se que todos teneis una parte deperezosos y estas cosas os gustan. Os enseñaré ahora algunas de las cosas que puedo hacer.

Ahora no me apetece. ¿No podemos hacer otra cosa?

por favor necesito que lo montes ahora

Ahora que me lo has pedido por favor, te haré caso. Me pongo a ello.

Figura 2: Conversación Alexa-UR3 en el reto 1.

Se crearon tablas en DynamoDB (tabla 1 y tabla 2) que contenían variables que se modificaban tanto desde la parte de Alexa como desde el propio UR, y que permitían la interacción con el robot.

\begin{tabular}{|l|l|}
\hline Nombre & Descripción \\
\hline $\mathrm{X}$ & Aquí se muestran los valores \\
$\mathrm{Y}$ & exactos de la posición de las \\
& $\begin{array}{l}\text { diferentes piezas en los ejes } \\
\text { cartesianos en milímetros. }\end{array}$ \\
\hline
\end{tabular}

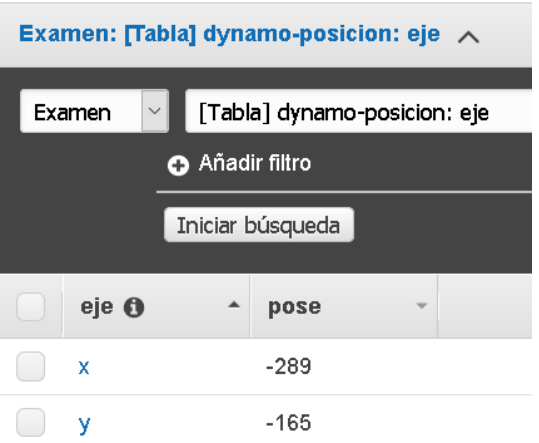

Tabla 1: DynamoDB posición de piezas

\begin{tabular}{|l|l|}
\hline Nombre & Descripción \\
\hline M1 & Estas variables son equivalentes a \\
M2 & $\begin{array}{l}\text { los diferentes retos, en los cuales } \\
\text { se le asigna un valor a cada } \\
\text { movimiento. }\end{array}$ \\
\hline
\end{tabular}

Examen: [Tabla] dynamo-maneja-robot: telefonoModel

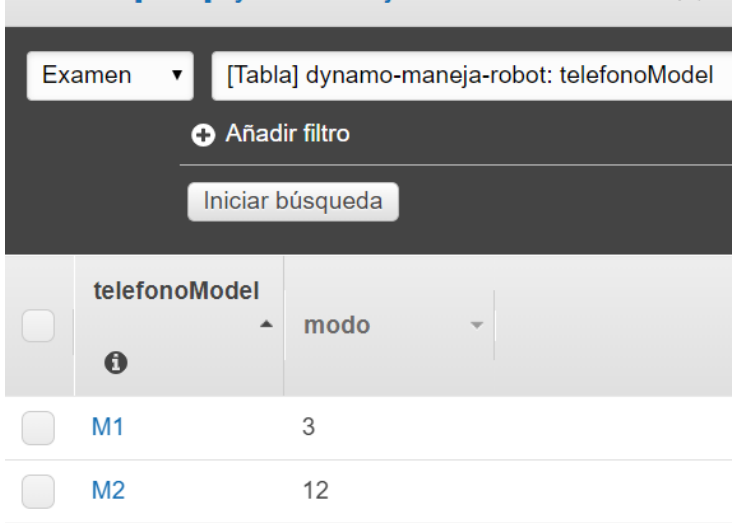

Tabla 2: Tabla de variables en las BBDD utilizada

\subsection{RETO 3}

El tercer reto es de Realidad Aumentada, AR. Las aplicaciones de AR realizadas en el Hackaton fueron dos:

- Muestra de los diferentes componentes internos de teléfonos móviles que tenemos en una base de datos, por medio de una animación del explotado del teléfono móvil, Figura 3. La imagen es tomada por una cámara Logitech c270 con la que se reconocerá el modelo de móvil. Se usa el motor de desarrollo Unity, por medio de su lenguaje de programación $\mathrm{C \#}$ y la librería Vuforia conseguiremos comunicarnos con la base de datos DynamoDB. La Figura 4 muestra parte del dialogo con el asistente de voz Alexa, que usaremos para el control de la animación. 


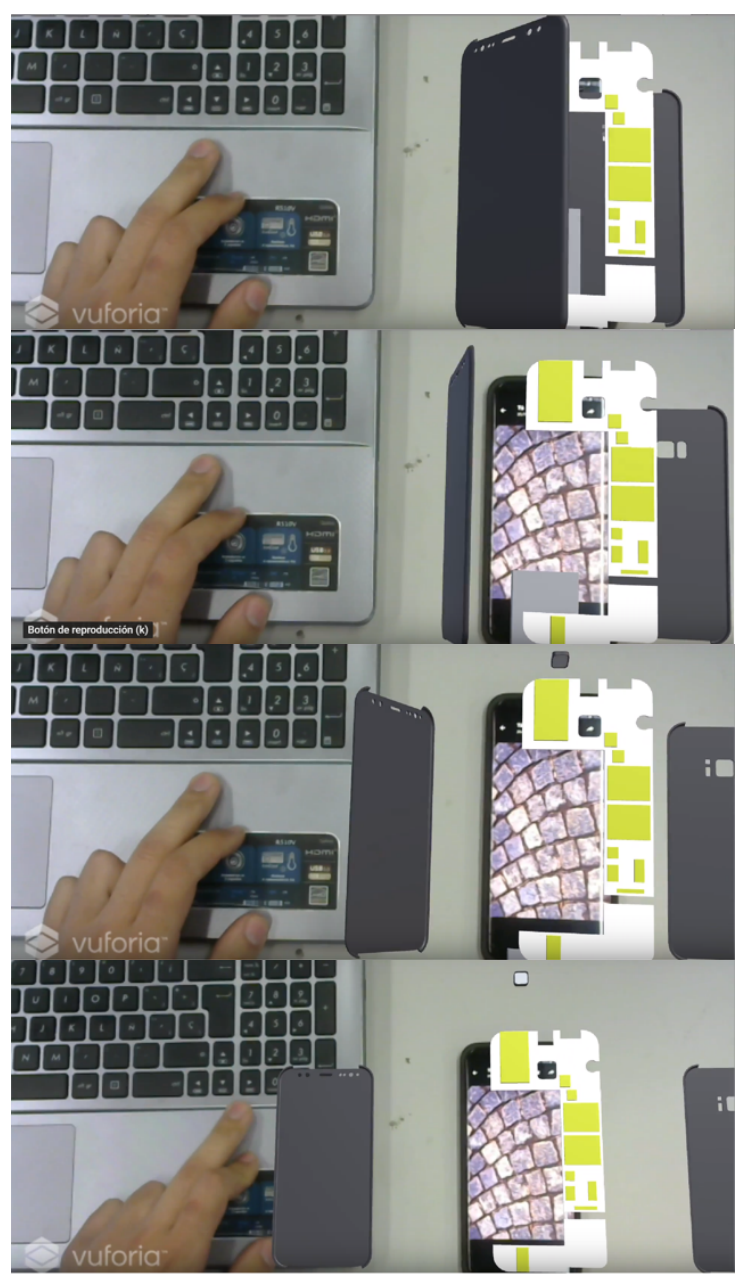

Figura 3: Animación explotado, Unity.

por último haz el explotado del móvil

perfecto. Voy a ello. Esta parte es la que más me gusta. Se puede ver como es el móvil por dentro y como lo he ido montando.

Figura 4: Conversación Alexa-UR3 en el reto 3.

- Reconocimiento, mediante la cámara Logitech c270, las diferentes piezas del móvil y su posición en el espacio, para su ensamblaje a posteriori. El lenguaje de programación utilizado para esta aplicación es Python que junto a la librería OpenCV podremos reconocer mediante un barrido del efector final del cobot, donde está situada la cámara, las diferentes piezas del móvil. La diferenciación y posición de las piezas se hace por un código RGB, cada pieza tendrá una zona con un área y color diferente, los datos de la posición de las piezas se pasarán a la base de datos DynamoDB, Tabla 1, base de datos que usará el robot para ir a las diferentes posiciones de las piezas. En esta parte nos conectaremos al asistente de voz Alexa, para poder interactuar con el robot y así poder hacer el barrido y ensamblaje del móvil. La Figura 5. Muestra una imagen del robot realizando el reto.

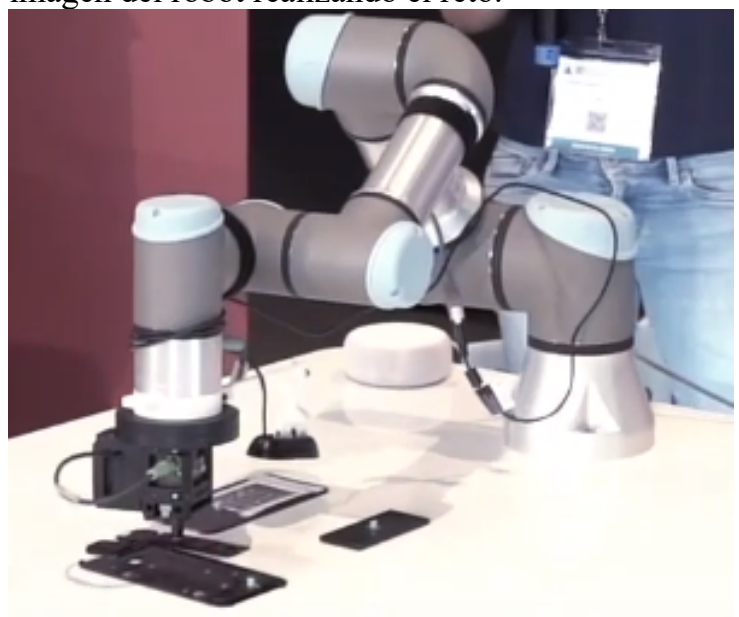

Figura 5: Montaje de piezas.

Este ejemplo de integración puede parametrizarse para ser utilizado en otros proyectos, usando como plantilla el esquema presentado y modificando los valores de las bases de datos, la conversación con Alexa y los movimientos del brazo robótico.

En resumen, un total de 10 alumnos de grado de las distintas titulaciones de la Escuela Politécnica Superior de Elche participaron durante 3 meses en la preparación de los retos, y 4 de ellos se desplazaron a Barcelona para la presentación del trabajo.

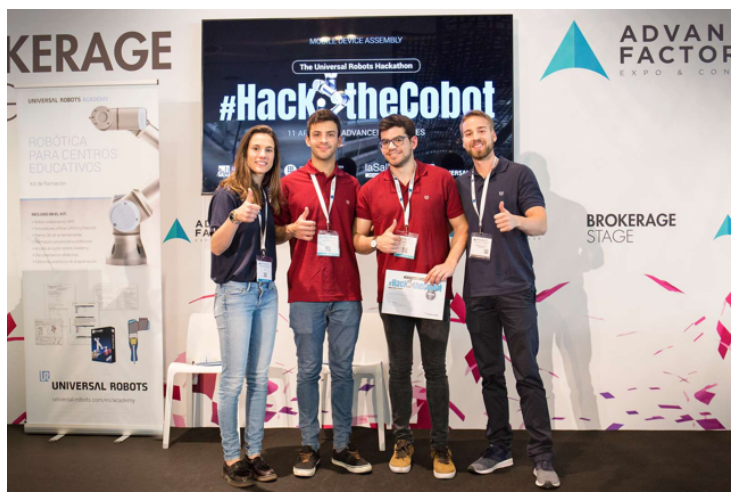

Figura 7: Foto del grupo en $\mathrm{BCN}$.

\section{Agradecimientos}

Los autores quieren agradecer el apoyo económico a la EPSE-UMH, al proyecto "Craneeal" - Referencia: DPI2016-80391-C3-2-R [AEI/FEDER, UE], financiado por la Agencia Estatal de Investigación (AEI) y por la Unión Europea a través del Fondo Europeo de Desarrollo Regional - FEDER - "Una 
manera de hacer Europa". José M. Vicente agradece a la Conselleria Educació, Investigació, Cultura i Esport de la Generalitat Valenciana su apoyo a través de FDEGENT/2018/015.

\section{English summary}

\section{EXAMPLE OF INTEGRATION OF ALEXA WITH A UR ROBOT}

\begin{abstract}
This work shows the solution adopted by a group of robotics students to allow a natural interaction with a robot using an intelligent loudspeaker and interactive cloud services. This solution allows to integrate within an industrial robot advanced options of voice interaction or $D B$ management with remote display of robot variables.
\end{abstract}

Keywords: Alexa, voice interaction, intelligent interaction.

\section{Referencias}

[1] "Virtual Assistant Platform" D Kužnar, A Tavčar, J Zupančič, M Duguleana. - 2016.

[2] "Un enfoque aplicado al control inteligente" $\mathrm{M}$. Santos - 2011.

[3] Gartner Inc., "Cloud Strategy Leadership," Gartner Inc., 2017. [Online]. Available: https://www.gartner.com/imagesrv/books/cloud/ cloud_strategy_leadership.pdf. [Accessed: May. 28, 2019].

[4] Microsoft Corporation, "Microsoft - Official Home Page," Microsoft Corporation, 2019. [Online].

Available: https://www.microsoft.com. [Accessed: May. 29, 2019].

[5] Amazon Inc., “Amazon Web Services (AWS) Cloud Computing Services", Amazon Inc., 2019. [Online]. Available: https://aws.amazon.com. [Accessed: May. 29, 2019].
[6] Dell EMC, "Data Storage, Cloud, Converged and Data Protection", Dell EMC, 2019. [Online]. Available: https://www.dellemc.com. [Accessed: May. 29, 2019].

[7] International Business Machines Corporation, "", International Business Machines Corporation, 2019. [Online]. Available: https://www.ibm.com. [Accessed: May. 29, 2019].

[8] Amazon Inc., "Amazon Alexa", Amazon Inc., 2019. [Online]. Available: https://developer.amazon.com/alexa. [Accessed: May. 29, 2019].

[9] Amazon Inc., "Alexa Skills Kit - Build for Voice with Amazon Alexa", Amazon Inc., 2019. [Online]. Available: https://developer.amazon.com/alexa-skillskit. [Accessed: May. 29, 2019.]

[10] Amazon Inc., "Alexa Voice Service - Make Your Own Alexa Product", Amazon Inc., 2019. [Online]. Available: https://developer.amazon.com/alexa-voiceservice. [Accessed: May. 29, 2019].

[11] Amazon Inc., "Amazon DynamoDB Overview”, Amazon Inc., 2019. [Online]. Available:

https://aws.amazon.com/dynamodb/?ncl=f 1 s. [Accessed: May. 29, 2019].

[12] Amazon Inc., "AWS Lambda - Serveless Compute - Amazon Web Services", Amazon Inc., 2019. [Online]. Available: https://aws.amazon.com/lambda. [Accessed: May. 29, 2019].

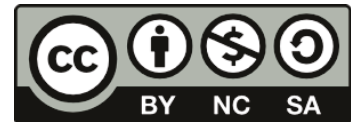

(C) 2019 by the authors. Submitted for possible open access publication under the terms and conditions of the Creative Commons Attribution CC BY-NC-SA 4.0 license (https://creativecommons.org/licenses/bync-sa/4.0/deed.es). 